\title{
Shock compression of polyurethane foams
}

\author{
D.M. Dattelbaum ${ }^{1}$, D.L. Robbins ${ }^{1}$, R.L. Gustavsen ${ }^{1}$, S.A. Sheffield ${ }^{1}$, D.B. Stahl ${ }^{1}$, \\ and J.D. $\mathrm{Coe}^{2}$
}

${ }^{1}$ Shock and Detonation Physics, Los Alamos National Laboratory, Los Alamos, NM 87545, USA
${ }^{2}$ Theoretical Division, Los Alamos National Laboratory, Los Alamos, NM 87545, USA

\begin{abstract}
Several shock studies have been made on polyurethane materials, both fully dense and distended in the form of foams. However, there is a lack of shock data between the densities of 0.321 and $1.264 \mathrm{~g} / \mathrm{cm}^{3}$ (fully dense). We present here data obtained from two different types of shock experiments at densities of $0.35,0.5,0.68,0.78$, and $0.9 \mathrm{~g} / \mathrm{cm}^{3}$ in order to fill in the density deficiencies and make it easier to develop an unreacted equation of state (EOS) for polyurethane as a function of density. A thermodynamically consistent EOS was developed, based on the Helmholtz free energy, and was used to predict the shock properties of polyurethane materials at densities from 1.264 to $0.348 \mathrm{~g} / \mathrm{cm}^{3}$. These estimates are compared to the available data. The data match quite close to the predictions and provide a basis for calculating polyurethane foam shock processes. Chemical reaction has been observed at relatively high pressure $(21.7 \mathrm{GPa})$ in fully dense polyurethane in an earlier study, and the equation of state presented here is representative of the unreacted polyurethane foam. Lowering the density is expected to drop the shock pressure for chemical reaction, yet there is not enough data to address the low density shock reaction thresholds in this study.
\end{abstract}

\section{Introduction}

Polyurethane foams are a common class of polymeric foams used extensively as structural components and shock mitigating materials. As such, there have been several previous studies focused on their impact or shock compression response and dynamic compaction behaviours. However, earlier studies have been limited to a few low initial densities as well as fully dense polyurethane. Here, we present new Hugoniot data for polymethylene diisocyanate (PMDI)-based polyurethane foam at a range of initial densities designed to fill in the gap in literature shock data in the density regime between 0.35 and $0.9 \mathrm{~g} / \mathrm{cm}^{3}$. We also present a thermodynamically consistent, unreacted equation of state that incorporates compaction and adequately represents the Hugoniot data obtained.

Hugoniot data for polyurethane materials are given in the Los Alamos Shock Compendium [1], a Los Alamos report [2], and a High Velocity Impact Phenomena book [3]. Fully dense polyurethane at a density of $1.264 \mathrm{~g} / \mathrm{cm}^{3}$ was well studied with the data indicating a chemical (or physical) reaction occurring at $21.7 \mathrm{GPa}$. The nature of this reaction is unknown but was conjectured to be due to chemical reaction by Carter and Marsh [2]. In addition to this fully dense data, there are data on 3 different initial densities of polyurethane foam; 0.321, 0.28, and $0.159 \mathrm{~g} / \mathrm{cm}^{3}$. All these data were obtained were obtained using explosively-driven assemblies, in which the shock transit times through multiple samples on top of a drive plate were measured using pins and/or streak cameras. [3] Equation-of-state standard samples co-located on the drive plate during the experiment were used to quantify the shock-input condition. The Hugoniot states were determined by impedance matching methods using the sample initial density, and shock velocity measurements. It was not possible to measure shock wave profiles in these experiments. The lower density foam Hugoniot data have few points with significant scatter within the data for the individual densities because of the difficulties in interpreting the flash-gap data.

There are several other earlier reports describing the dynamic response of polyurethane foams. Kipp et al. examined the shock compression and release response of polyurethane foam (density of $0.22 \mathrm{~g} / \mathrm{cm}^{3}$ ) in very low pressure, gas-gun driven experiments. [4] The pressures ranged from 0.36 to $0.6 \mathrm{GPa}$, much lower than the experiments of interest in this study. Baer [5] and Skews [6] studied very low density polyurethane foam at very low shock pressures that are not relevant to this study. Maw and Whitworth [7] did one explosively driven shot on $\sim 0.3 \mathrm{~g} / \mathrm{cm}^{3}$ polyurethane foam and further examined historical data using a Lagrangian hydrocode.

\section{Experimental details}

\subsection{Materials}

PMDI-based polyurethane foam samples were prepared with initial densities of (nominally) $\rho$ g $0.35,0.5,0.68$, 0.78 , and $0.9 \mathrm{~g} / \mathrm{cm}^{3}$ in rectangular test blocks. The foam samples were light beige in color and contained average pore sizes (diameters) of $\sim 75$ and $240 \mu \mathrm{m}$ by $\mathrm{X}$-ray computed tomography. Foam samples for the shock experiments were machined from the center of the test blocks.

\subsection{Plate impact experiments}

Two types of plate impact experiments were performed on foam samples, all driven by a large bore two stage light gas gun with a launch tube bore diameter of $50 \mathrm{~mm}$, and maximum velocity of $3.6 \mathrm{~km} / \mathrm{s}$, described previously. [8] Projectile velocities were measured using a laser diode 


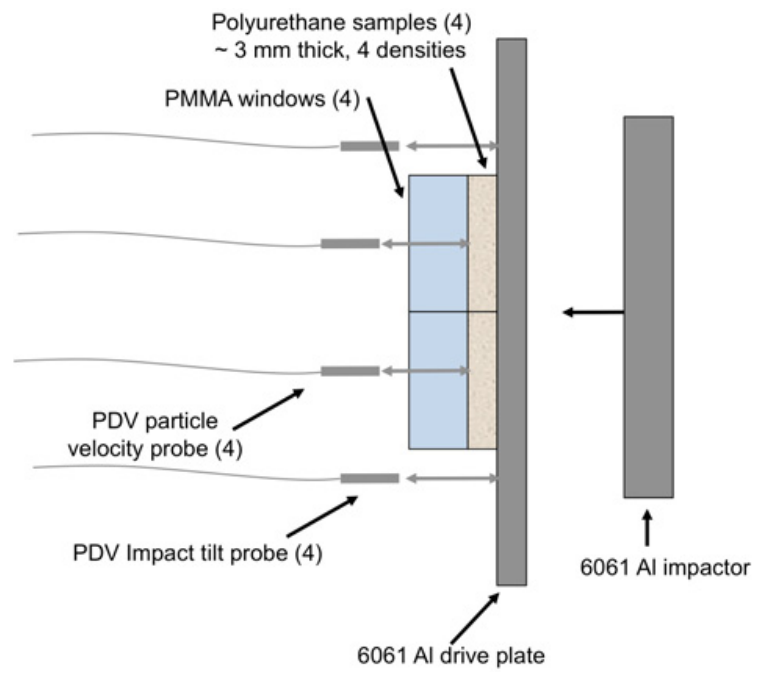

Fig. 1. Schematic illustration of the multi-slug experimental target assembly. Four foam samples of different initial densities are glued to the rear surface of an $6061 \mathrm{Al}$ drive plate. Each sample is approximately $3 \mathrm{~mm}$ thick. The samples are backed by PMMA windows containing an $\mathrm{Al}$ mirror at the interface for particle velocity measurements. Eight PDV probes are used in the experiment: 4 for interface particle velocity and timeof-arrival measurements at the polyurethane-PMMA interface, and 4 at the rear of the drive plate for shock arrival and tilt correction.

system mounted at the exit interface of launch tube. The drop in voltage at 4 photodiode detectors associated with the edge of the projectile clipping the 4 laser beams was recorded just prior to impact. Accuracy of the projectile velocity measurement is about 2 to $5 \mathrm{~m} / \mathrm{s}$.

The first type of experiment, referred to as a front surface impact (or reverse ballistic geometry), consists of a foam sample affixed to the front of a Lexan projectile which impacts an oriented single-crystal LiF window mounted just beyond the end of the two-stage launch barrel. A copper disk was mounted behind the foam to reach off-Hugoniot states in the ring-up that occurs after the initial shock; this will not be discussed in this paper. Eight $\mathrm{k} \AA$ of aluminum was coated on the LiF window, which had been roughened so that a diffuse mirror was produced on the $\mathrm{LiF}$ impact surface. In many of the experiments, this mirror was further protected using a $1 \mathrm{~mm}$-thick oriented single-crystal LiF cover because the foam rapidly destroyed the thin mirror on impact. Dual velocity interferometers called VISARs [9] were fielded on each experiment using the protected mirror to obtain the interface particle velocity wave profile. The measured particle velocity at the interface, and the projectile velocity were used to determine the principal Hugoniot state using shock impedance methods.

The second type of experiment, referred to as a "multislug" experimental configuration, is a shock transmission experiment. The multi-slug configuration, adapted to our two-stage gun, was first applied by Gustavsen [10] and is shown schematically in figure 1 . Four foam samples were mounted to a $6061 \mathrm{Al}$ drive plate. A PMMA window (with $8 \mathrm{k} \AA$ vapor-coated mirrors on the surface in
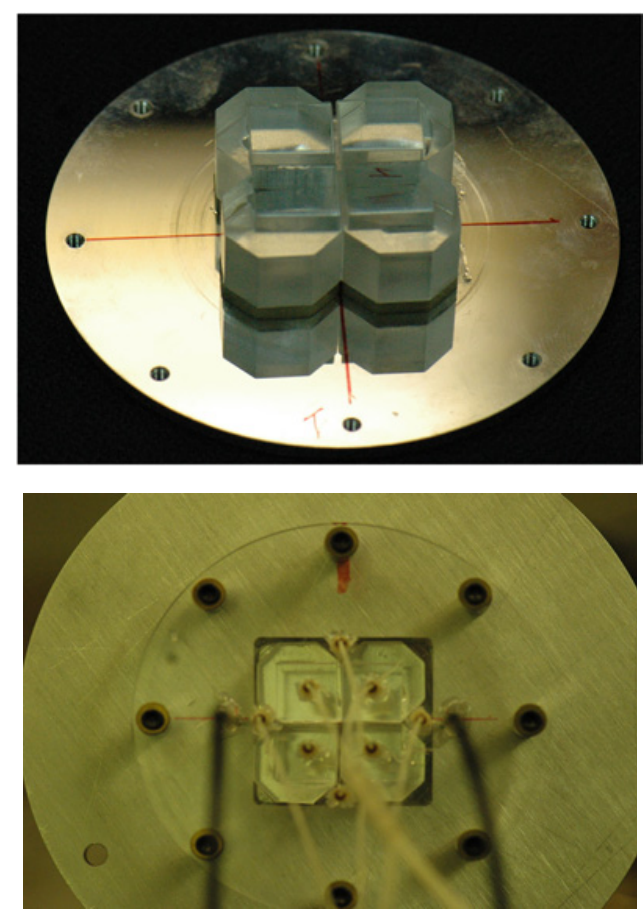

Fig. 2. Photographs of a multi-slug target assembly. (Top) individual foam samples with PMMA windows glued to the Al baseplate. (Bottom) Rear view of a fully-assembled target showing the 4 foam samples with 4 PDV probes. There are also 4 PDV probes at the outside intersection of the samples to measure the free surface velocity of the $\mathrm{Al}$ drive plate.

contact with the foam) was mounted on the rear surface of each foam sample. The interface particle history at each sample/PMMA interface was measured using Photon Doppler Velocimetry (PDV). [11] Each sample had a PDV probe at the rear interface with PMMA, and there were 4 additional probes measuring the free-surface velocity of the Al plate (and providing a measure of projectile tilt), making a total of 8 PDV measurements on each experiment. Photographs of a multi-slug target during and after assembly are shown in Fig. 2.

The multi-slug target was mounted just beyond the end of the two-stage gun launch tube where it was impacted by a 6061 Al plate mounted in a Lexan projectile.

A well-defined, supported shock wave travels through the $\mathrm{Al}$ drive plate and into the foam samples producing a flat-topped shock which interacts with the PMMA window. The 8 PDV interferometer systems measure the time the shock exits the Al plate (enters the foam samples) and the time (and interface velocity) the shock breaks out from the foam samples into the PMMA windows. This information provides the average shock velocity $\left(U_{s}\right)$ through each foam sample and the interface particle velocity $\left(u_{p}\right)$ at the sample/PMMA interface. The shock arrival times were corrected for system timing and projectile tilt. Hugoniot data were determined by impedance matching using the equation of state for $6061 \mathrm{Al}\left(\rho_{0}=2.703 \mathrm{~g} / \mathrm{cm}^{3}, \mathrm{c}_{0}=\right.$ $5.288, \mathrm{~s}=1.3756$, where $\left.\mathrm{U}_{\mathrm{s}}=\mathrm{c}_{0}+\mathrm{s} \mathrm{u}_{\mathrm{p}}\right)$, the measured projectile velocity, initial density, and shock velocity through the foam. 
Table 1. Results of 3 front surface impact experiments. Error in the Hugoniot parameters is estimated to be $<2 \%$.

\begin{tabular}{|c|c|c|c|c|c|c|}
\hline $\begin{array}{l}\text { Shot } \\
\text { No. }\end{array}$ & $\begin{array}{l}\text { Init. } \\
\text { Den. } \\
\mathrm{g} / \mathrm{cm}^{3}\end{array}$ & $\begin{array}{c}\text { Proj. } \\
\text { Vel. } \\
\mathrm{km} / \mathrm{s}\end{array}$ & $\begin{array}{c}\mathrm{U}_{\mathrm{s}} \\
\mathrm{mm} / \mu \mathrm{s}\end{array}$ & $\begin{array}{c}\mathrm{u}_{\mathrm{p}} \\
\mathrm{mm} / \mu \mathrm{s}\end{array}$ & $\begin{array}{c}\text { Press. } \\
\mathrm{GPa}\end{array}$ & $\mathrm{V} / \mathrm{V}_{0}$ \\
\hline $2 S-410$ & 0.687 & 2.148 & 3.18 & 1.868 & 4.08 & 0.413 \\
\hline $2 S-426$ & 0.676 & 2.986 & 4.012 & 2.534 & 6.87 & 0.368 \\
\hline $2 S-452$ & 0.957 & 2.118 & 3.974 & 1.692 & 6.43 & 0.574 \\
\hline
\end{tabular}

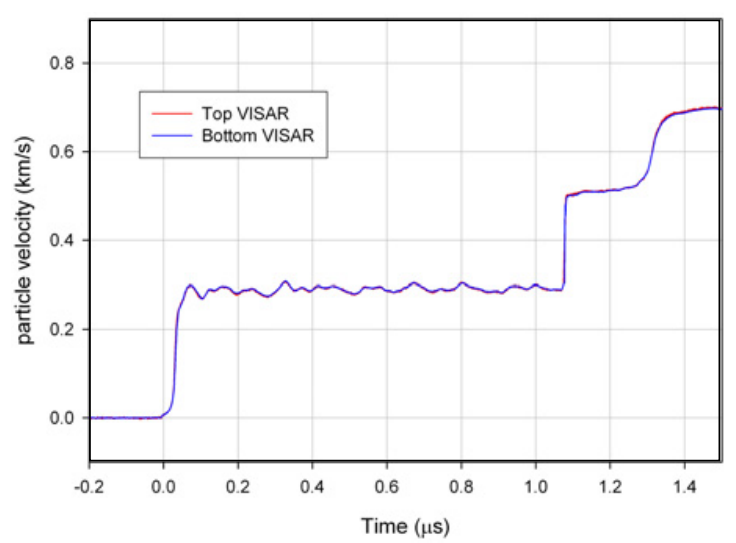

Fig. 3. Particle velocity wave profiles from shot $2 S-410$ recorded using dual VISARs at the foam-LiF interface (the two measurements are essentially on top of each other). The second and subsequent shocks are due to shock reverberations between the $\mathrm{LiF}$ target window and a copper disk mounted behind the foam in the projectile. Analysis of them is not part of this study.

\section{Results}

Three front surface impact experiments were completed at densities of 0.68 and 0.96 polyurethane foam densities. Three multi-slug experiments were completed with 4 different density foam samples on each one. Shot $2 \mathrm{~S}$ 531 had samples with densities of $0.87,0.67,0.63$, and $0.49 \mathrm{~g} / \mathrm{cm}^{3}$. Shot $2 \mathrm{~S}-555$ had samples of $0.87,0.63,0.49$, and $0.35 \mathrm{~g} / \mathrm{cm}^{3}$. Shot $2 \mathrm{~S}-556$ had samples of $0.87,0.63$, 0.49 , and $0.35 \mathrm{~g} / \mathrm{cm}^{3}$. From all these experiments, there were 1 new Hugoniot point for $0.96 \mathrm{~g} / \mathrm{cm}^{3}, 3$ for $0.87 \mathrm{~g} / \mathrm{cm}^{3}, 3$ for $0.67(9) \mathrm{g} / \mathrm{cm}^{3}, 3$ for $0.63 \mathrm{~g} / \mathrm{cm}^{3}, 3$ for $0.49 \mathrm{~g} / \mathrm{cm}^{3}$, and 2 for $0.35 \mathrm{~g} / \mathrm{cm}^{3}$, making a total of 15 new Hugoniot points for polyurethane foam in this study.

\subsection{Front surface impact experiments}

Table 1 summarizes the results of the 3 front surface impact experiments performed on polyurethane foam at intermediate densities $\rho=0.687 \mathrm{~g} / \mathrm{cm}^{3}$ and $0.957 \mathrm{~g} / \mathrm{cm}^{3}$. The foam samples were found to have a thin, high density skin on the surfaces of the test blocks. To avoid measurement of the properties of the skin in the plate impact experiments, the samples were machined from below the surface of the foam blocks.

Hugoniot states were determined using the measured projectile velocity and interface particle velocity obtained from the dual VISAR measurements, and the LiF equation

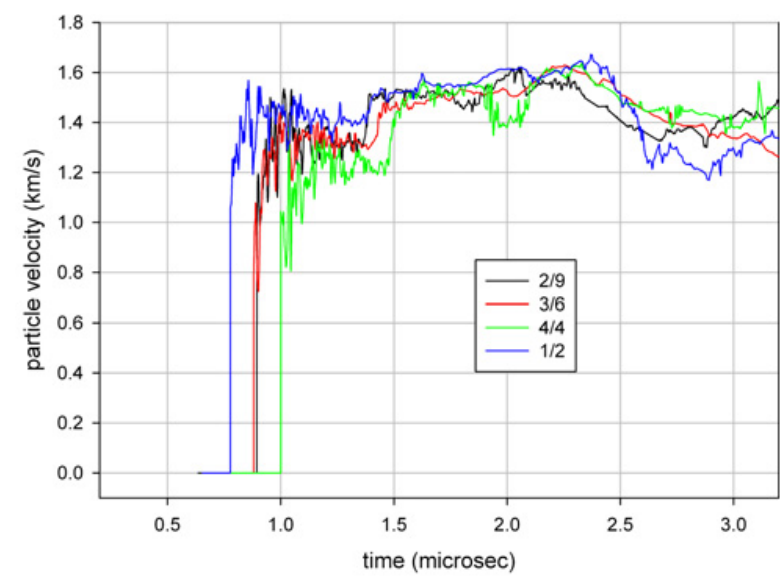

Fig. 4. Particle velocity profiles measured using PDV at the polyurethane foam/PMMA interface from shot 2S-531. The traces correspond to samples with $\rho_{0}=0.868 \mathrm{~g} / \mathrm{cm}^{3}$ (blue), $\rho_{0}=0.670 \mathrm{~g} / \mathrm{cm}^{3}$ (red), $\rho_{0}=0.626 \mathrm{~g} / \mathrm{cm}^{3}$ (black), and $\rho_{0}=0.489 \mathrm{~g} / \mathrm{cm}^{3}$ (green). Note the increase in transit time, and decrease in peak particle velocity as the density decreases.

of state $\left(\rho_{0}=2.638 \mathrm{~g} / \mathrm{cm}^{3}, \mathrm{c}_{0}=5.15, \mathrm{~s}=1.35\right.$ where $\left.\mathrm{U}_{\mathrm{s}}=\mathrm{c}_{0}+\mathrm{su}_{\mathrm{p}}\right)$ in impedance match calculations. The remaining Hugoniot variables in Table 1 were calculated using the conservation equations. [3]

A representative interface particle velocity wave profile obtained in shot $2 \mathrm{~S}-410$ is shown in figure 3 . The VISAR probe collects the Doppler-shifted laser light from an area $\sim 0.5 \mathrm{~mm}$ in diameter, resulting in an averaging of the interface velocities over this area. Even so, there is evidence of structure in the first wave of the shock wave profile shown in figure 3; this is due to the heterogeneous structure of the foam sample. There is also evidence of some dispersion (wave-front rounding) in the front of the profile due to the foam porosity.

\subsection{Multi-slug experiments}

Three multi-slug experiments were performed with foam samples ranging in density from $0.348-0.868 \mathrm{~g} / \mathrm{cm}^{3}$. The resulting 12 Hugoniot points are summarized in Table 2. The pressure of the Hugoniot state in each foam sample differs in the same experiment because it depends on the density of the sample. For example, in shot $2 \mathrm{~S}-566$, the projectile velocity of $2.624 \mathrm{~km} / \mathrm{s}$ results in shock input pressures of $2.99 \mathrm{GPa}$ for $\rho_{0}=0.348 \mathrm{~g} / \mathrm{cm}^{3}$ to $8.08 \mathrm{GPa}$ for $\rho_{0}=0.868 \mathrm{~g} / \mathrm{cm}^{3}$.

Differences between the foam samples is also readily observed in the particle velocity profiles recorded at the polyurethane-PMMA interfaces measured using PDV. Figure 4 shows the particle velocity profiles for the 4 foam samples measured at the foam/PMMA interfaces from shot 2S-531. The sample densities and corresponding trace colors are: $\rho_{0}=0.868 \mathrm{~g} / \mathrm{cm}^{3}$ (blue), $\rho_{0}=0.670 \mathrm{~g} / \mathrm{cm}^{3}$ (red), $\rho_{0}=0.626 \mathrm{~g} / \mathrm{cm}^{3}$ (black), $\rho_{0}=0.489 \mathrm{~g} / \mathrm{cm}^{3}$ (green). The shock transit times are progressively longer as the density decreases across the series. In addition, the particle velocity at the interface is lower with decreasing density. The shock wave profiles show complex structure, which is 
Table 2. Summary of Hugoniot data obtained from the multi-slug experiments.

\begin{tabular}{|l|c|c|c|c|c|c|}
\hline Shot No. & Proj. vel. $(\mathrm{km} / \mathrm{s})$ & Initial density $\left(\mathrm{g} / \mathrm{cm}^{3}\right)$ & Impactor & $\mathrm{U}_{\mathrm{s}}(\mu \mathrm{m} / \mathrm{ms})$ & $\mathrm{u}_{\mathrm{p}}(\mu \mathrm{m} / \mathrm{ms})$ & $\mathrm{P}(\mathrm{GPa})$ \\
\hline $2 \mathrm{~S}-531$ & $2.046 \pm 0.0004$ & $0.868 \pm 0.01$ & $6061 \mathrm{Al}$ & $3.861 \pm 0.028$ & $1.685 \pm 0.004$ & $5.66 \pm 0.06$ \\
\hline $2 \mathrm{~S}-531$ & $2.046 \pm 0.0004$ & $0.670 \pm 0.01$ & $6061 \mathrm{Al}$ & $3.346 \pm 0.022$ & $1.784 \pm 0.004$ & $4.00 \pm 0.06$ \\
\hline $2 \mathrm{~S}-531$ & $2.046 \pm 0.0004$ & $0.626 \pm 0.01$ & $6061 \mathrm{Al}$ & $3.404 \pm 0.022$ & $1.795 \pm 0.004$ & $3.83 \pm 0.06$ \\
\hline $2 \mathrm{~S}-531$ & $2.046 \pm 0.0004$ & $0.489 \pm 0.01$ & $6061 \mathrm{Al}$ & $3.004 \pm 0.018$ & $1.863 \pm 0.003$ & $2.74 \pm 0.05$ \\
\hline $2 \mathrm{~S}-555$ & $1.215 \pm 0.037$ & $0.868 \pm 0.01$ & $6061 \mathrm{Al}$ & $2.470 \pm 0.080$ & $1.062 \pm 0.033$ & $2.28 \pm 0.10$ \\
\hline $2 \mathrm{~S}-555$ & $1.215 \pm 0.037$ & $0.626 \pm 0.01$ & $6061 \mathrm{Al}$ & $1.991 \pm 0.053$ & $1.120 \pm 0.034$ & $1.40 \pm 0.03$ \\
\hline $2 \mathrm{~S}-555$ & $1.215 \pm 0.037$ & $0.489 \pm 0.01$ & $6061 \mathrm{Al}$ & $1.822 \pm 0.045$ & $1.145 \pm 0.035$ & $1.02 \pm 0.06$ \\
\hline $2 \mathrm{~S}-555$ & $1.215 \pm 0.037$ & $0.348 \pm 0.01$ & $6061 \mathrm{Al}$ & $1.746 \pm 0.040$ & $1.166 \pm 0.036$ & $0.79 \pm 0.03$ \\
\hline $2 \mathrm{~S}-556$ & $2.624 \pm 0.001$ & $0.868 \pm 0.01$ & $6061 \mathrm{Al}$ & $4.383 \pm 0.015$ & $2.124 \pm 0.004$ & $8.08 \pm 0.08$ \\
\hline $2 \mathrm{~S}-556$ & $2.624 \pm 0.001$ & $0.626 \pm 0.01$ & $6061 \mathrm{Al}$ & $3.825 \pm 0.013$ & $2.275 \pm 0.004$ & $5.45 \pm 0.08$ \\
\hline $2 \mathrm{~S}-556$ & $2.624 \pm 0.001$ & $0.489 \pm 0.01$ & $6061 \mathrm{Al}$ & $3.752 \pm 0.012$ & $2.344 \pm 0.005$ & $4.30 \pm 0.08$ \\
\hline $2 \mathrm{~S}-556$ & $2.624 \pm 0.001$ & $0.348 \pm 0.01$ & $6061 \mathrm{Al}$ & $3.543 \pm 0.012$ & $2.425 \pm 0.005$ & $2.99 \pm 0.08$ \\
\hline
\end{tabular}

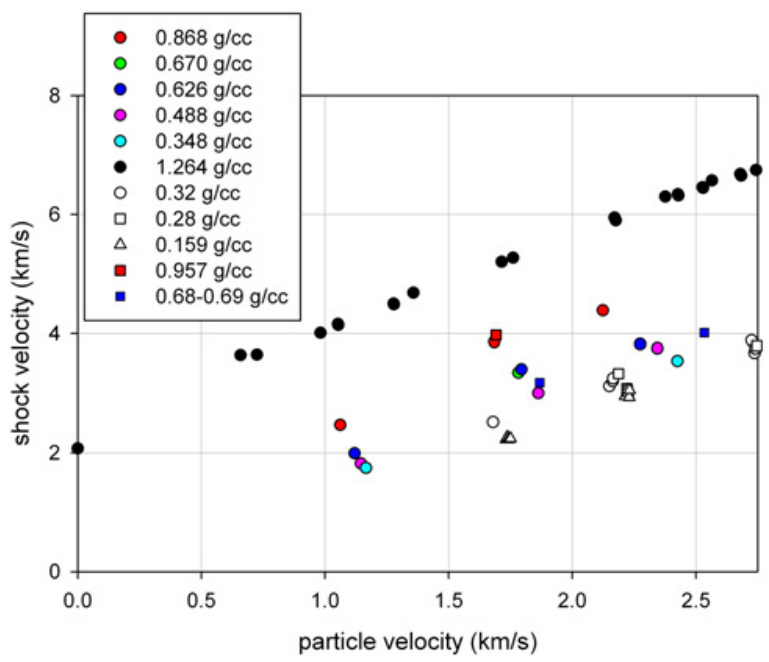

Fig. 5. Summary of Hugoniot data for polyurethane with initial densities from 0.159 to $1.264 \mathrm{~g} / \mathrm{cm}^{3}$ in the shock velocity $\left(\mathrm{U}_{\mathrm{s}}\right)$ particle velocity $\left(\mathrm{u}_{\mathrm{p}}\right)$ plane from Los Alamos historical data and this work. The new data, shown in the colored symbols, fill the gap in the historical Hugoniot data at intermediate densities.

due to the porosity (heterogeneous microstructure) of the foam samples.

The Hugoniot data from this work are overlaid with the Los Alamos historical data in the shock velocityparticle velocity, and pressure-volume planes in figures 5 and 6, respectively. The high density Marsh Hugoniot defines the dynamic response of the solid, near-TMD polyurethane. At low shock input strengths $(2.3 \mathrm{GPa}$ for example), the $\rho_{0}=0.868 \mathrm{~g} / \mathrm{cm}^{3}$ foam appears to be fully compacted since they are near the fully dense data in figure 6. Incomplete compaction is evident for the lower density foams, particularly for the 0.489 and $0.348 \mathrm{~g} / \mathrm{cm}^{3}$ materials. There is significant scatter in the data for the

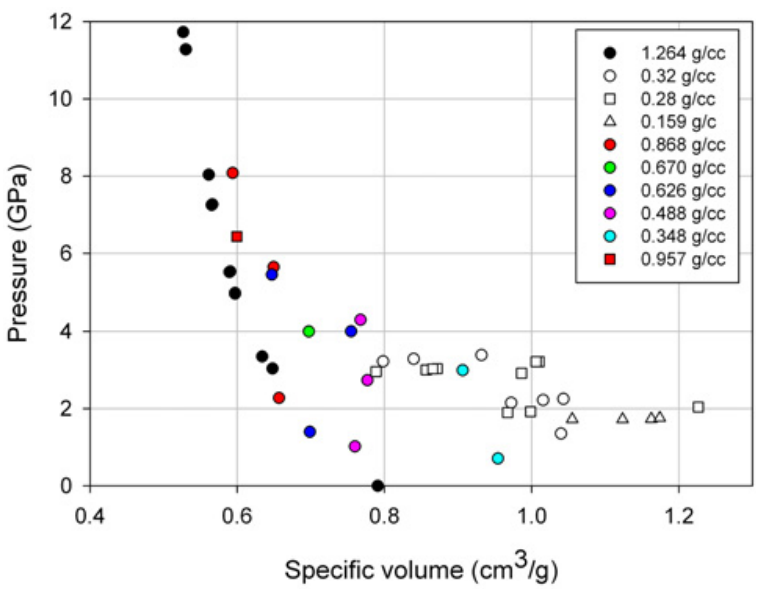

Fig. 6. Hugoniot data for polyurethane from Los Alamos historical data and this work in the pressure $(\mathrm{P})$-specific volume $(\mathrm{V})$ plane. The high density Hugoniot defines the dynamic compression of fully dense polyurethane. There is considerable scatter in the low-to-intermediate density foam Hugoniot data.

low density materials, consistent with the historical data, because of the difficulty of making good measurements on these heterogeneous materials.

Another aspect of these measurements is the possibility of chemical reaction. As alluded to in the introduction, fully dense polyurethane has a cusp in the Hugoniot at about $21.7 \mathrm{GPa}$ which is assumed to be due to chemical reaction. As the density decreases the amount of compression work by the initial shock increases dramatically, causing the sample temperature to also increase dramatically. Because of this, chemical reaction would be expected at much lower input pressures. However, the number of experiments completed to this point is insufficient to understand where reaction takes place at each density. 


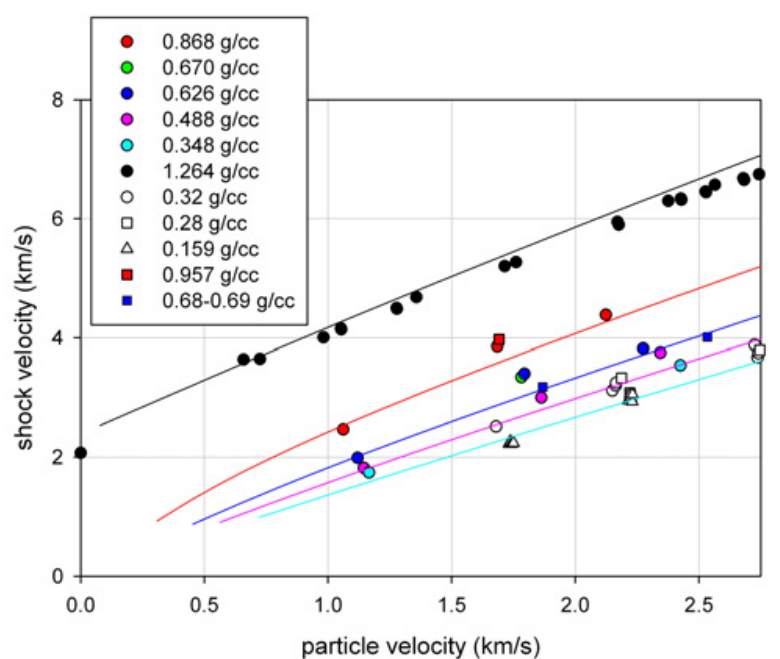

Fig. 7. Hugoniot data derived from Marsh and new LANL experiments for polyurethane foam at a range of initial densities (symbols), overlaid calculated Hugoniots (solid lines) from a Hayes EOS with a P- $\alpha$ model. Note that the unreacted EOS does not account for chemical reaction in the foam.

Additional experiments will be necessary to address this area.

\section{Discussion}

In order to be able to model polyurethane foam shock experiments, it was necessary to develop an unreacted EOS, which would allow prediction of the shock properties of the foam materials at a variety of initial densities. A thermodynamically consistent EOS based on the Helmholtz free energy [12] and applied in concert with the $\mathrm{P}-\alpha$ formalism for distended materials [13]. This procedure allows us to estimate the Hugoniot behavior or fully dense and porous polyurethane of varying initial densities. The form of the equation of state, referred to as a Hayes EOS, is given in Eq. 1.

$$
\begin{aligned}
F(T, V)= & C_{v}\left[\left(T-T_{0}\left(1+\frac{\Gamma}{V}\left(V_{0}-V\right)\right)+T \log \left(\frac{T_{0}}{T}\right)\right]\right. \\
& +\left(\frac{K_{T, 0} V_{0}}{N(N-1)}\right)\left[\left(\frac{V_{0}}{V}\right)^{N-1}-(N-1)\left(1-\frac{V}{V_{0}}\right)\right]
\end{aligned}
$$

where $T$ is temperature, $V$ is specific volume, $\Gamma$ is the Grüneisen parameter, $K_{T, 0}$ is the isothermal compressibility, $C_{v}$ is the specific heat at constant volume, $F$ is the Helmholtz free energy, and $N$ is a constant. In this EOS form, both the heat capacity and ratio of the Grüneisen parameter/volume $(\Gamma / \mathrm{V})$ are assumed to be constants. A Murnaghan EOS fit to the full density $\left(1.264 \mathrm{~g} / \mathrm{cm}^{3}\right)$ polyurethane Hugoniot data in the low pressure regime was used to estimate the zero-pressure isothermal bulk modulus (assuming little deviation between the isotherm, isentrope, and Hugoniot at very low compressions) and its pressure derivative, $N$ according to:

$$
P=\frac{K_{T, 0}}{N}\left[\left(\frac{V_{0}}{V}\right)^{N}-1\right]
$$

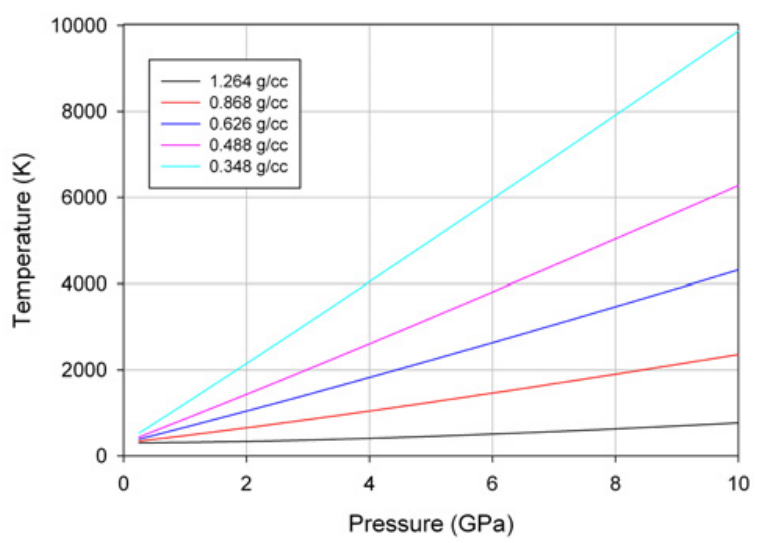

Fig. 8. Temperature $(\mathrm{K})$ as a function of shock pressure on the principal Hugoniot for 5 densities of polyurethane foam, calculated using the Helmholtz-based equation of state.

where $P$ is the pressure in GPa. From the fit, $K_{T, 0} \sim$ 7.22 GPa, and $\mathrm{N}=5.8$. Using an estimate for the Grüneisen parameter of $\Gamma / \mathrm{V}=0.5$, the Hugoniot curves in figure 7 were computed for densities of 1.264 (black), 0.78 (orange), 0.68 (purple), 0.5 (pink), and 0.35 (light blue) $\mathrm{g} / \mathrm{cm}^{3}$. The calculated Hugoniots compare well with the fully dense data (black filled circles) and the lower density data from the Los Alamos historical data. This method of developing an EOS does a reasonable job at capturing the density dependence of the shock compressive response in the. An improved EOS could be developed by using better estimates for the heat capacity and the Grüneisen parameter, and establishing their temperature dependence rather than being constant.

It should be noted that this unreacted EOS does not account for chemical reaction of the polyurethane, which occurs at an input pressure of $21.7 \mathrm{GPa}$ in the fully dense polyurethane. It likely occurs at much lower input pressures in the foam (depending on density) because of the large amount of compression energy (and subsequent temperature increase) that is produced in the shock. To give some idea of the high temperatures involved as the initial density decreases, figure 8 is a plot of stress vs. calculated temperature for several densities of polyurethane.

In summary, unreacted Hugoniot data for polyurethane at several initial densities has been obtained for a PMDIbased polyurethane foam. The data were obtained using two different gas-gun driven plate impact experiments; front surface impacts where the polyurethane was impacted on a window and multi-slug experiments where 4 different density samples were shocked and the transmitted waves measured. All measurements were made with laserbased interferometry, either VISAR or PDV. The data fill critical phase space between $\rho_{0}=0.348-1.264 \mathrm{~g} / \mathrm{cm}^{3}$, where there was a previous lack of data. Below $\rho_{0} \sim$ $0.5 \mathrm{~g} / \mathrm{cm}^{3}$, there is evidence of incomplete compaction of the foam over the range of shock pressures studied to date. Additional experiments should focus on two regimes: the low pressure regime in which the foams are still crushing up, and high pressure experiments on low densities foams to investigate anomalous compaction phenomena common in porous materials. 
An unreacted EOS based on the Helmholtz free energy and a P- $\alpha$ compaction model (referred to as a Hayes EOS) was calibrated to match the measured data at the different densities. Both the Los Alamos historical data and the new data of this study were used in the EOS development process. The EOS predicts Hugoniots that adequately overlay the experimental data points for the different densities. These predictions can be used in modeling shock processes involving polyurethane within the unreacted regime.

\section{Acknowledgements}

We acknowledge funding from DOE/NNSA for this work. Los Alamos National Laboratory is operated by LANS LLC for the Department of Energy. We also thank Brian Bartram, Adam Pacheco, Lee Gibson and Ben Hollowell for help in firing the gas guns at Chamber 9 .

\section{References}

1. LASL Shock Hugoniot Data, S.P. Marsh (Ed.) University of California, (1980).

2. S.P. Marsh, W.J. Carter Hugoniot equation of state of polymers, Los Alamos National Laboratory report LA-13006-MS (1995).

3. R.G. McQueen, S.P. Marsh, J.W. Taylor, J.N. Fritz, W.J. Carter, The equation of state of solids from shock wave studies, in High Velocity Impact Phenomena, R. Kinslow (Ed.), chapter 7, Academic Press, NY, NY (1970).
4. M.E. Kipp, L.C. Chhabildas, W.D. Reinhart, M.K. Wong, Shock Compression of Condensed Matter1999, M.D. Furnish, L.C. Chhabildas, R.S. Hixson (Eds.), AIP Conference Proceedings 505, p. 313 (1999).

5. M.R. Baer, Shock Waves, 2, 121 (1992).

6. B.W. Skews, Shock Waves, 1, 205 (1991).

7. J.R. Maw, N.J. Whitworth, R.B. Holland, Shock Compression of Condensed Matter-1995, S.C. Schmidt, W.C. Tao (Eds.), AIP Conference Proceedings 370, p. 133 (1995).

8. R.R. Alcon, S.A. Sheffield, A.R. Martinez, R.L. Gustavsen, Shock Compression of Condensed Matter1997, S.C. Schmidt, D.P. Dandekar, J.W. Forbes (Eds.), AIP Conference Proceedings 429, p. 845 (1997).

9. L.M. Barker, R.E. Hollenbach, J. Appl. Phys. 41, 4208-4226 (1970).

10. R.L. Gustavsen, D.G. Thompson, B.W. Olinger, R. Deluca, B.D. Bartram, T.H. Pierce, Proceedings of the $14^{\text {th }}$ Inernationl Deonation Symposium, Office of Naval Research Report ONR-351-10-185, p. 655 (2010).

11. O.T. Strand, D.R. Goosman, C. Martinez, T.L. Whitworth and W.W. Kuhlow, Rev. Sci. Inst. 77, 083108 (2006).

12. S.A. Sheffield, D.E. Mitchell, D.B. Hayes, Proeedings of the $6^{\text {th }}$ Symposium (International) on Detonation, Office of Naval Research Report ACR-221, p. 748 (1976).

13. W. Herrmann, J. Appl. Phys. 40, 2490 (1969). 